\title{
L'acca ballerina e la grafia delle interiezioni
}

\author{
Enrico Testa
}

PUBBLICATO: 30 GIUGNO 2019

$\Omega$ ra i tanti punti critici dell'uso scritto dell'italiano in rapporto o in attrito con il suo uso parlato, largo spazio s'è dato ad alcuni grandi, importanti fenomeni, genericamente radunabili sotto le categorie della semplificazione e dell'estensione analogica: istituti morfosintattici e testuali che, dalla sorte del congiuntivo al sistema dei pronomi, tanto hanno fatto discutere specialisti e parlanti sensibili ai problemi della lingua. Minore attenzione ha ricevuto, di fronte a questi fondamentali e, per certi versi, incanutiti personaggi della nostra scena linguistica, una riottosa tribù di monelli verbali: le interiezioni, manifestazioni dirette della voce, della presenza dell'enunciatore e del suo stato d'animo. In quanto richiamano l'attenzione dell'ascoltatore (o del lettore o del personaggio di un dialogo letterario), vennero paragonate da Leo Spitzer, nel suo Lingua italiana del dialogo del I922, a "squilli di tromba": esse "sono come musica assoluta, come canti senza parole, riflesso melodico dei moti interiori, con cui si fanno presagire le sfumature del discorso e si prepara l'ascoltatore all'atmosfera di quanto sarà detto" (Spitzer 2007, p. 66-67). E assai prima - era il I772 - il filosofo e letterato tedesco Johann Gottfried Herder, nello splendido Saggio sullorigine del linguaggio, pensava che in questi suoni "molto semplici", ora sospiro ora urlo, risiedesse il linguaggio originario, naturale e affettivo, non artefatto e addirittura condiviso con altre specie; e, a parer suo, nel loro passaggio alla lingua più elaborata, nel loro tramutarsi in segni, si poteva ancora sentir vibrare remotissimi "accenti naturali" (Herder 1995, p. 33-34).

Tanti anni sono passati dall'epoca in cui le idee di Herder ebbero corso (anche se sono sopravvissute, a vari gradi di consapevolezza e in diverse misure, nella scrittura di alcuni poeti: da Pascoli a Caproni); e il pensiero linguistico moderno ha calcato più sul valore pragmatico e culturale di questi fenomeni (emblematico il fatto che interiezioni caratteristiche di una lingua non ricorrano in una lingua anche storicamente vicina) che su presunti tratti originari o, addirittura, 'universali' (una convinzione a cui potevano indurre la semplicità di forme come ah o uh o, ancor più, le espressioni onomatopeiche).

Parole cosi umili ed esili e talvolta indisciplinate, si classificano come parole invariabili e costituiscono il solo tipo di categoria lessicale in grado di trasmettere il significato di un'intera frase. Un aspetto, questo, particolarmente evidente quando l'interiezione ricorre in posizione isolata costituendo da sola un'intera battuta dello scambio dialogico; ma la loro collocazione può darsi sia all'inizio, con la funzione segnalata da Spitzer, che alla fine o all'interno di frasi con effetti espressivi particolarmente interessanti (Nencioni I977: 233-235). Sono caratteristiche soprattutto della dimensione parlata, e informale, della lingua e si distinguono in due categorie: primarie o proprie con solo valore interiettivo, prive di significato lessicale e dal senso variabile in relazione al contesto (tra le più frequenti: ah, uh, eh); e secondarie o improprie, parti del discorso appartenenti al sistema lessicale (nomi, aggettivi, avverbi, verbi) usate però in funzione interiettiva sino ad assumere il valore di frase intera (Poggi I995, p. 4I3), come peccato!, bravo!, bene!, guarda!

Come si può vedere scorrendo gli esempi appena fatti, il tratto rilevante nella resa grafica delle interiezioni primarie è dato dall'occorrenza del segno, o grafema diacritico, $h$; che, in italiano, non ha valore fonetico ma esclusivamente ortografico (è detta appunto $h$ muta). Le sue principali funzioni sono quelle di indicare la pronuncia velare di c e $g$ davanti a $i$ ed e (chitarra, ghiro); e di differenziare le forme del presente indicativo di avere (ho, hai, ha, hanno) da termini con la stessa pronuncia (o, ai, a, 
anno). L'h può però rappresentare un suono, "ma solo come realizzazione facoltativa" (Serianni ig89, p. 44 ), quando compare in alcune interiezioni primarie. In tal caso puo indicare un'aspirazione (o, forse meglio, un tono sospirato o esitante). E allora, accanto alla "pronuncia usuale di $a h$, eh, ehm e simili ([a], $[\varepsilon]$ o $[\mathrm{e}],[\mathrm{em}]$, più o meno prolungati), è possibile sentire occasionalmente, specie in caso d'iterazione, [ha], [he], [he], [hem]". Inoltre, le interiezioni primarie possono, sempre occasionalmente, contenere suoni estranei al nostro sistema fonologico. Ad esempio, öh esprime conferma mentre öoh sorpresa o indignazione, "entrambe pronunciate con vocale anteriore medio-alta, fonema che non appartiene al sistema italiano" (Cignetti 20I0, p. 672 e Poggi I995: 4I4); e il cosiddetto click, suono prodotto facendo schioccare la lingua contro il palato o contro i denti, presente in lingue remote (soprattutto africane), ricorre, approssimativamente rappresentabile come ts, nella nostra per esprimere perplessità o negazione (o, ripetuto, come segno di richiamo indirizzato ai gatti).

Se queste forme e oscillazioni dipendono dall'iridescente varietà della voce, non è forse però opportuno, allorché si prova a dar segno nello scritto di tonalità e significati di uninterazione parlata percorsa da questi fenomeni, ricorrere a scelte arbitrarie che possano dar luogo a confusione e fraintendimenti. D'altronde, il repertorio del parlato-scritto confezionato dai dialoghi teatrali e narrativi, offre gran parte di quanto è necessario. Così, la lunghezza della e distingue tra èh come segno di conferma ed éeh come segno di esitante reticenza, mentre eh!eh! può, già in Manzoni, esprimere, con largo spettro di soluzioni interpuntive, sorpresa o rimprovero (Testa I997, p. 43).

Il problema della trascrizione delle interiezioni primarie si è fatto via via più forte con l'aumento della pressione dell'oralità sulla scrittura e, in modo particolare, sullitaliano digitato: testi dei blog, e-mail, sms. Ma è particolarmente sentito anche dagli aspiranti romanzieri alle prese con la costruzione di dialoghi che vorrebbero essere vivacemente mimetici dell'oralità. A tal punto che siti che si propongono di assicurare il successo letterario ai loro utenti, dedicano non poco spazio alla questione.

Il punto dolente si può, in sintesi, isolare nella domanda "ma l'acca dove va?". È il grafema diacritico per eccellenza dell'italiano che s'e fatto ballerino sull'odierna scena della scrittura delle interiezioni primarie. Capita in tal modo di incontrare - contravvenendo all'uso e alla norma che prevedono l'h in posizione finale o all'interno di parola - invece di ahi, boh, beh, ah!, ahimé, mah, oh!, ehi, rispettivamente le seguenti forme: hai, ai, bho, bhe, ha!, haimè, mha, ho! e o!, ei. Le ragioni per privilegiare la prima serie rispetto alla seconda è che con la prima è possibile distinguere l'interiezione da una parola omofona (preposizione semplice e articolata, congiunzione, forma del verbo avere) evitando equivoci e cadute di 'ruolo', o prestigio, linguistico, per quanto questo possa ancora valer oggi... Che poi ci siano margini di libertà e creatività è indubbio: oh può dilatarsi in ohh per dar rilievo alla meraviglia o brr farsi, come nei fumetti, brrr con un profluvio di vocali e consonanti che talvolta rischia però di essere eccessivo e manierato (che è poi il risultato antitetico all'espressione immediata e 'istintiva' di uno stato soggettivo del parlante).

Un secondo buon motivo per prestare attenzione alla grafia dei fenomeni interiettivi, affidandosi alle rese canoniche, è dato dal fatto che quest'ultime si sono consolidate attraverso i nostri maestri della prosa narrativa e teatrale allorché si sono misurati, nei dialoghi ma non solo, con il parlato-scritto: non si vede perché si debba, in proposito, arbitrariamente violare il repertorio definito negli ultimi due secoli da Manzoni, Nievo, Pirandello, Pavese, Fenoglio, Sciascia, Calvino, Levi o Natalia Ginzburg.

In terzo luogo, se è vero - come s'è visto - che le interiezioni utilizzano anche suoni che non fanno parte del sistema fonologico italiano, non sembra questa una ragione sufficiente per adottare soluzioni 'mimetiche' di altre lingue e realtà fonologiche. È, ad esempio, particolarmente diffusa, 
nell'italiano digitato e altrove, la trascrizione della risata con hahaha al posto del tradizionale ahahah. La prima sequenza, anche se la forma ha ha come espressione d'ironia e di sarcasmo ha delle attestazioni già nel Cinquecento, pare essersi diffusa per influsso dell'inglese e se ne motiva l'adozione in quanto meglio imiterebbe il suono aspirato che accompagna la risata. L'articolazione aspirata della $h$ in principio di parola però in italiano non si dà mentre è fonema presente in molte altre lingue, tra cui il tedesco e, appunto, l'inglese. Allora perché cambiare? Per moda linguistica? Perché più vicina al suono effettivo di una risata? Difficile dirlo se si pensa a come ognuno di noi rida, in fondo, in maniere e con 'melodie' diverse. Di fronte a tale pluralità di realizzazioni, impossibile a rendersi nello scritto, forse è più opportuno servirsi di una grafia convenzionale. Anche perché - ma questo dipende dalla particolare sensibilità del parlante - la sequenza hahaha pare più una litania della terza persona singolare del verbo avere (quasi un'epigrafe rappresentativa di Paperon de' Paperoni) che una libera espressione della voce.

Insomma, per quanto debole - sottoposto com'è ai diversi eventi dell'emotività e al plurimo intonarsi delle voci - il sistema delle interiezioni una sua grammatica ce l'ha. $\mathrm{O}$, meglio, sul piano della loro trascrizione grafica, che rimarrà sempre incostante e infedele rispetto all'esecuzione parlata, regole e convenzioni esistono da tempo, consegnateci soprattutto dagli autori che non hanno rinunciato a riprodurre, con i pochi arnesi a loro disposizione, le sfuggenti manifestazioni di una realtà cosi volatile come quella dell'oralità. Una grammatica minima e particolare, certo, che va però appresa al pari di quella che riguarda gli aspetti del linguaggio articolato. Se, come scriveva Gramsci nella lettera del i 8 maggio I93I alla moglie Giulia, la grammatica è "una frazione della vita" (Gramsci i965, p. 566), è altrettanto importante, considerata quanta parte occupano, nella vita, affettività e sentimenti, essere in grado di dar conto nello scritto delle loro insorgenze. E farlo in una maniera tale che possa "essere intesa e condivisa da tutti e da ciascuno" (Herder i995, p. 32).

Nota bibliografica:

- Bazzanella I994: Carla Bazzanella, Le facce del parlare, Firenze, La Nuova Italia, 1994

- Cignetti 20ro: Luca Cignetti, interiezione, in Simone, R., diretta da, Enciclopedia dellitaliano, Roma, Treccani, 20I0, pp. 67I-674

- Ehlich 1986: Konrad Ehlich, Interjektionen, Tübingen, Niemeyer, 1986

- Gramsci r965: Antonio Gramsci, Lettere dal carcere, Torino, Einaudi, 1965

- Herder i995: Johann Gottfried Herder, Saggio sullorigine del linguaggio (I772), Parma, Pratiche Editrice, I995

- Nencioni 1983: Giovanni Nencioni, L'interiezione nel dialogo teatrale di Pirandello (1977), in Tra grammatica e retorica, Torino, Einaudi, 1983 , pp. 210-253

- Poggi i995: Isabella Poggi, Le interiezioni, in Renzi, A. - Salvi, G. - Cardinaletti, A., a cura di, Grande grammatica italiana di consultazione, Bologna, il Mulino, vol. III, I995, pp. 403-425

- Serianni 1989: Luca Serianni, Grammatica italiana. Italiano comune e lingua letteraria, con la collaborazione di A. Castelvecchi, Torino, Utet, 1989

- Spitzer 2007: Leo Spitzer, Lingua italiana del dialogo (I922), Milano, il Saggiatore, 2007

- Testa r997: Enrico Testa, Lo stile semplice. Discorso e romanzo, Torino, Einaudi, I997 


\section{Cita come:}

Enrico Testa, L'acca ballerina e la grafia delle interiezioni , "Italiano digitale", 2019, IX, 2019/2 (aprile-giugno)

DOI: $10.35948 / 2532-9006 / 2020.3236$

Copyright 2019 Accademia della Crusca

Pubblicato con licenza creative commons CC BY-NC-ND 